\title{
Retardance and flicker modeling and characterization of electro-optic linear retarders by averaged Stokes polarimetry
}

\author{
Francisco J. Martínez, ${ }^{1,2}$ Andrés Márquez, ${ }^{1,2, *}$ Sergi Gallego, ${ }^{1,2}$ Jorge Francés, ${ }^{1,2}$ \\ Inmaculada Pascual, ${ }^{2,3}$ and Augusto Beléndez ${ }^{1,2}$ \\ ${ }^{1}$ Dept. de Física, Ing. de Sistemas y Teoría de la Señal, Universidad de Alicante, Ap. 99, E-03080 Alicante, Spain \\ ${ }^{2}$ I.U. Física Aplicada a las Ciencias y las Tecnologías Universidad de Alicante, Ap. 99, E-03080 Alicante, Spain \\ ${ }^{3}$ Dept. de Óptica, Farmacología y Anatomía, Universidad de Alicante, Ap. 99, E-03080 Alicante, Spain \\ ${ }^{*}$ Corresponding author: andres.marquez@ua.es
}

Received October 16, 2013; revised December 22, 2013; accepted January 12, 2014;

posted January 14, 2014 (Doc. ID 199630); published February 12, 2014

\begin{abstract}
A polarimetric method for the measurement of linear retardance in the presence of phase fluctuations is presented. This can be applied to electro-optic devices behaving as variable linear retarders. The method is based on an extended Mueller matrix model for the linear retarder containing the time-averaged effects of the instabilities. As a result, an averaged Stokes polarimetry technique is proposed to characterize both the retardance and its flicker magnitude. Predictive capability of the approach is experimentally demonstrated, validating the model and the calibration technique. The approach is applied to liquid crystal on silicon displays (LCoS) using a commercial Stokes polarimeter. Both the magnitude of the average retardance and the amplitude of its fluctuation are obtained for each gray level value addressed, thus enabling a complete phase characterization of the LCoS. @ 2014 Optical Society of America

OCIS codes: (120.2040) Displays; (120.5410) Polarimetry; (230.3720) Liquid-crystal devices; (230.6120) Spatial light modulators; (230.2090) Electro-optical devices.

http://dx.doi.org/10.1364/OL.39.001011
\end{abstract}

The linear variable retarder is a common electro-optical device that may be used to generate or to detect specific states of polarization (SOP) in systems such as Stokes or Mueller matrix polarimeters [1-3]. More complex devices, such as parallel aligned liquid crystal on silicon (PA-LCoS) displays $[4,5]$ or devices such as the liquid crystal-based point diffraction interferometer proposed by Ramirez et al. [6], can be assimilated to linear variable retarders. Since these electro-optic devices are totally characterized by their linear retardance values, then methods typically used in the characterization of waveplates become available. The most popular are ellipsometric, polarimetric, and interferometric ones [1, 7$]$.

One property common to all linear retardance characterization methods is that they assume that the birefringence in the waveplate has a constant value, no fluctuations, during the measurement process. In the presence of fluctuations or instabilities, measured for example in modern LCoS devices [8-13] these methods may provide erroneous results [14]. Furthermore, the amplitude of the retardance fluctuation becomes a magnitude of interest for a more accurate characterization and modeling of the device under test. Recently some techniques have been demonstrated by our group [14] and by Ramirez et al. [15], based respectively on the classical linear polarimeter and in a combination of linear and circular polarimeters.

In the present Letter, we provide a more general framework to analyze the existence of fluctuations in the retardance of variable linear retarders, which may be directly applied to the widely used PA-LCoS devices. We use the Mueller-Stokes formalism and through this analysis we demonstrate a straightforward calibration technique, based on a conventional Stokes polarimeter, to measure the average retardance and the magnitude of its fluctuation amplitude as a function of the applied voltage. We further demonstrate the predictive capability of this theoretical framework and its associated calibration methodology. First, we will introduce the theoretical framework. Then, average retardance and flicker will be experimentally calibrated for a commercially available PA-LCoS, using a commercial rotating waveplate Stokes polarimeter. Eventually, predictive capability of our proposal will be demonstrated by comparing experimental and simulated results.

The methodology that we propose is based on the Mueller-Stokes formalism [1] , which enables one to deal both with polarized and with unpolarized light. A number of electro-optic devices, such as PA-LCoS displays, can be modeled as a variable retardance waveplate, whose linear retardance $\Gamma$ depends on the voltage applied. Specifically, the Mueller matrix $M_{R}(\Gamma)$ of a linear retarder with a retardance value $\Gamma$, with its fast axis along the $x$ axis, is given by

$$
M_{R}(\Gamma)=\left(\begin{array}{cccc}
1 & 0 & 0 & 0 \\
0 & 1 & 0 & 0 \\
0 & 0 & \cos \Gamma & \sin \Gamma \\
0 & 0 & -\sin \Gamma & \cos \Gamma
\end{array}\right) .
$$

This well-known expression considers a constant value for the retardance. In order to incorporate the existence of fluctuations or instabilities, let us consider a triangular profile for the variation of retardance with time $\Gamma(t)$. This is a reasonable assumption in the case of PA-LCoS as can be seen from the instantaneous measurement plots shown in a number of papers [9,-14]. Furthermore, the triangular time-dependent profile represents actually a linear model, thus, the first option to 
try before more complex approaches may be proven necessary. The triangular profile can be analytically expressed by the following equation:

$$
\Gamma(t)=\left\{\begin{array}{cc}
\bar{\Gamma}-a+\frac{2 a}{T / 2} t & 0 \leq t<T / 2 \\
\bar{\Gamma}+3 a-\frac{2 a}{T / 2} t & T / 2 \leq t<T
\end{array}\right.
$$

where $\bar{\Gamma}$ and $a$ are respectively the values for the average retardance and its fluctuation amplitude, and $T$ is the period for the fluctuation instability.

Using the linear time-dependent fluctuation model we can calculate a more general expression for the Mueller matrix of the linear retarder. Typically fluctuations, if existent, are considerably faster than the integration time of detector systems; thus the expression of interest in most applications is the averaged Mueller matrix for the linear retarder. Thus, we need to calculate the average values for the cosine and the sine functions in Eq. (1):

$$
\langle\cos \Gamma(t)\rangle=(\sin a / a) \cos (\bar{\Gamma}),
$$

$$
\langle\sin \Gamma(t)\rangle=(\sin a / a) \sin (\bar{\Gamma}) .
$$

Taking into account Eqs. (3) and (4) we can describe our averaged matrix for the linear retarder as

$$
\left\langle M_{R}(\bar{\Gamma}, a)\right\rangle=\left(\begin{array}{cccc}
1 & 0 & 0 & 0 \\
0 & 1 & 0 & 0 \\
0 & 0 & (\sin a / a) \cos \bar{\Gamma} & (\sin a / a) \sin \bar{\Gamma} \\
0 & 0 & -(\sin a / a) \sin \bar{\Gamma} & (\sin a / a) \cos \bar{\Gamma}
\end{array}\right) .
$$

This expression provides a more realistic and precise model of the linear retarder, where the average retardance and its fluctuation need to be characterized.

Now we are interested in analyzing the output SOP, produced by a linear retarder fulfilling Eq. (5). Two different working geometries may be considered for electro-optic devices: in transmission and in reflection. In the latter case, an inversion of the horizontal axis is produced between the corresponding forward and the backward (right-handed) reference systems, which in the Mueller-Stokes formalism is expressed by the inversion matrix as follows:

$$
\operatorname{Inv}=\left(\begin{array}{cccc}
1 & 0 & 0 & 0 \\
0 & 1 & 0 & 0 \\
0 & 0 & -1 & 0 \\
0 & 0 & 0 & -1
\end{array}\right)
$$

Then, in the case of reflective devices, the averaged output SOP can be calculated as follows:

$$
\left\langle S_{\text {out }}\right\rangle=\operatorname{Inv} \cdot\left\langle M_{R}(\bar{\Gamma}, a)\right\rangle \cdot S_{\text {in }} .
$$

For transmissive devices, no inversion matrix needs to be considered. The resultant average Stokes vector is

$$
\left\langle S_{\text {out }}\right\rangle=\left(\begin{array}{c}
S_{0} \\
S_{1} \\
\pm(\sin a / a)\left(S_{2} \cos \bar{\Gamma}+S_{3} \sin \bar{\Gamma}\right) \\
\pm(\sin a / a)\left(-S_{2} \sin \bar{\Gamma}+S_{3} \cos \bar{\Gamma}\right)
\end{array}\right)
$$

where the Stokes components for the input SOP are $S_{0}$, $S_{1}, S_{2}$, and $S_{3}$. The plus and minus signs before the third and fourth Stokes components apply for transmissive and reflective devices respectively. Let us calculate the degree of polarization (DoP) for this average output SOP:

$$
\operatorname{DoP}=\sqrt{\left(S_{1}\right)^{2}+(\sin a / a)^{2}\left(\left(S_{2}\right)^{2}+\left(S_{3}\right)^{2}\right)} / S_{0} .
$$

We note that the DoP depends on the input SOP and on the amplitude $a$ of the fluctuations, but it does not depend on the average retardance $\bar{\Gamma}$. According to the model proposed, Eqs. (5), (8), and (9) provide all the information necessary to calculate the performance of the electro-optic device under test and the resulting output light wavefront.

In the rest of the Letter, to simplify the discussion, we will restrict our attention to reflective devices, which means that in Eq. (8) we have to consider the minus sign accompanying the third and fourth Stokes components. Extension to transmissive devices is then straightforward. Now let us take a closer look into Eqs. (8) and (9): We may find specific input SOPs that may prove useful to measure the two parameters in the model, $\bar{\Gamma}$ and $a$. In this sense, if the beam impinging the retarder corresponds to linearly polarized light at $+45^{\circ}$ with respect to the $X$ axis, i.e., $\left(S_{0}=1, S_{1}=0, S_{2}=1, S_{3}=0\right)$, the average SOP and DoP at the output will be expressed as follows:

$$
\begin{gathered}
\left\langle S_{\text {out }}\right\rangle=\left(\begin{array}{c}
1 \\
0 \\
-(\sin a / a) \cos \bar{\Gamma} \\
(\sin a / a) \sin \bar{\Gamma}
\end{array}\right), \\
D o P=(\sin a / a) .
\end{gathered}
$$

We note that the output $S_{1}$ component is zero independently of the retardance and its fluctuation amplitude. The expression for DoP is also straightforward and is directly related with the fluctuation amplitude. Equations (10) and (11) can be used to measure both the average retardance value $\overline{\bar{\Gamma}}$ and its fluctuation amplitude $a$. This can be easily accomplished using Eq. (11) to obtain the fluctuation amplitude $a$, and the ratio between the third and fourth Stokes vector components, i.e., $-\left\langle S_{3}\right\rangle /\left\langle S_{2}\right\rangle=\operatorname{tg}(\bar{\Gamma})$, to obtain $\bar{\Gamma}$.

The model and the accompanying calibration technique that we propose in this Letter may be applied to any device that can be modeled as a variable waveplate retarder. Specifically in this Letter, we analyze a commercial PA-LCoS display, model PLUTO distributed by the company HOLOEYE. It is a nematic liquid crystal filled, with $1920 \times 1080$ pixels and 0.7 inch diagonal. It is electronically configured to obtain a $360^{\circ}$ retardance range at the $633 \mathrm{~nm}$ wavelength. 
The polarimetric measurements have been obtained with a Stokes polarimeter, model PAX5710VIS-T distributed by the company THORLABS. This is a rotating waveplate-based polarimeter, which belongs to time-division mode polarimeters [16]. They are not able to provide reliable instantaneous values if the SOP is changing more rapidly than its measurement time interval. In particular, the polarimeter is able to provide the values for the Stokes vector after half a rotation of the waveplate, which corresponds to a measurement time interval of $3 \mathrm{~ms}$ (maximum rotation frequency is $333 \mathrm{~Hz}$ ). On the other hand, the software allows the measurement time interval to be enlarged to obtain an averaged signal. When enough rotations are considered, and if the periods of fluctuation and half-rotation are not multiple, then for each angular position of the rotating waveplate the amount of samples collected is representative of the time-varying SOP generated by the fluctuations in the device. Taking into account that the time period (frequency) for the fluctuations in our PA-LCoS device is $8.66 \mathrm{~ms}(120 \mathrm{~Hz})$, we have tried different averaging time-interval options with the polarimeter software. The polarimeter averaging time considered in the paper, $600 \mathrm{~ms}$, is much larger than actually needed to obtain fully stable and repeatable SOP measurements.

In Fig. 1 we show the average Stokes vector components and the DoP measured with the Stokes polarimeter as a function of the gray level value addressed onto the LCoS device. The LCoS setup includes an input polarizer with its transmission axis at $+45^{\circ}$ with respect to the laboratory vertical ( $x$ axis for the right-handed system used in this work). The unexpanded beam of a fully polarized $\mathrm{He}-\mathrm{Ne}$ laser impinges quasi-perpendicularly to the LCoS, at $3^{\circ}$ incidence angle to separate the input and the reflected beam. The director axis of the LCoS is along the horizontal. Typically, the director axis in nematic devices corresponds to the slow axis. From the results in Fig. 1 we note that parameter $S_{1}$ is close to zero in clear confirmation of the predicted result in Eq. (10). Values measured for DoP vary between 1.003 and 0.939 as gray levels increase. Some depolarized light may be produced by scattering or multiple reflections [8]. This was measured with the LCoS switched off and DoP values higher than 1 were obtained, so this origin for unpolarized can be disregarded in the present case.

In Fig. 2 , we plot the calculated average retardance and its fluctuation amplitude. We observe that the retardance range is about $360^{\circ}$ with a very good linearity. The

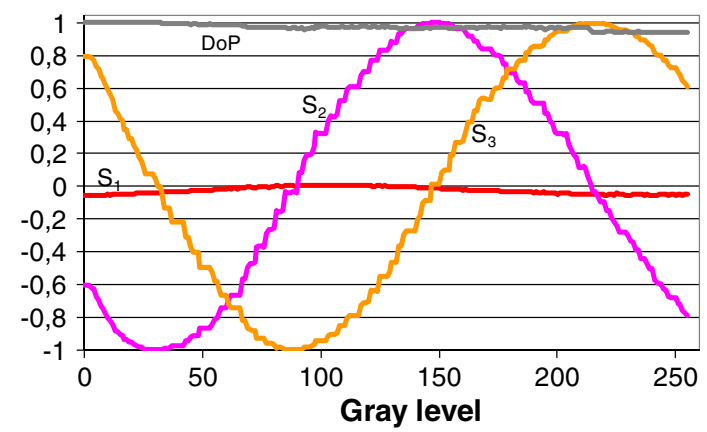

Fig. 1. Experimental values for the Stokes parameters and DoP, for input SOP linear at $+45^{\circ}$ and $\lambda=633 \mathrm{~nm}$.

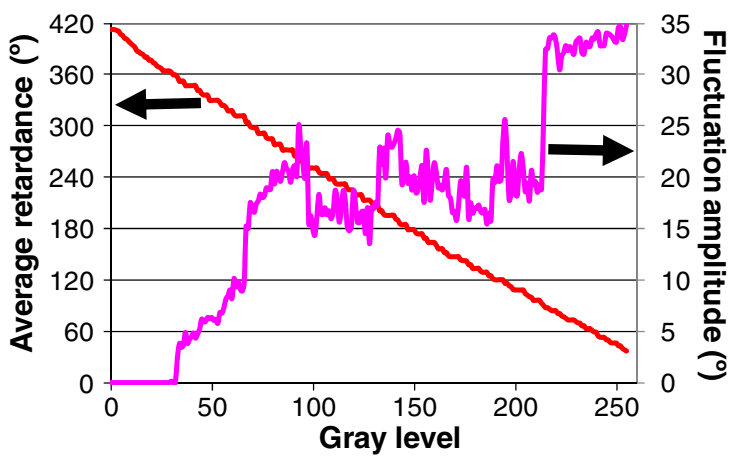

Fig. 2. Calculated values for the average retardance and the fluctuation amplitude for $\lambda=633 \mathrm{~nm}$.

fluctuation amplitude is close to $0^{\circ}$ for lower gray levels and increases to about $35^{\circ}$ for higher gray levels. The various jumps encountered in the fluctuation amplitude reveal the pulsed nature of the digital signal addressed onto the LCoS. For DoP > 1, nonphysical values, we have considered that fluctuation amplitude is $0^{\circ}$.

Once the retardance and its fluctuation magnitude have been calibrated, we can apply these values in Eqs. (5), (8), and (9) to predict the output Stokes vector for any input SOP. To this goal we have measured the reflected SOP for an incident light beam linearly polarized at $15^{\circ}$ with respect to the lab vertical. In Figs. 3 and 4 we plot respectively the normalized Stokes parameters and the DoP and both the experimental (dotted lines) and the simulated (continuous lines) values are compared.

We see that there is a very good agreement between the model and the experimental results. In Fig. $\underline{3}$ we note the constancy of the $S_{1}$ component and the oscillatory behavior of $S_{2}$ and $S_{3}$ components, in accordance with the expression in Eq. (8) where the retardance affects the third and fourth components through the cosine and sine functions. In Fig. $\underline{4}$ we also see how the model is able to predict the DoP with a good accuracy across the whole gray level range. This is a very important result since this proves the enhanced validity of the model proposed in Eq. (2) for the time fluctuations. Therefore, the calibration performed enables a full prediction of the average output SOP.

For a more complete analysis of the predictive capability of our model, we have also obtained measurements for a circularly polarized incident light beam. In particular, we have measured the reflected SOP for right-handed circularly polarized light, i.e., $\left(S_{0}=1, S_{1}=0, S_{2}=0\right.$,

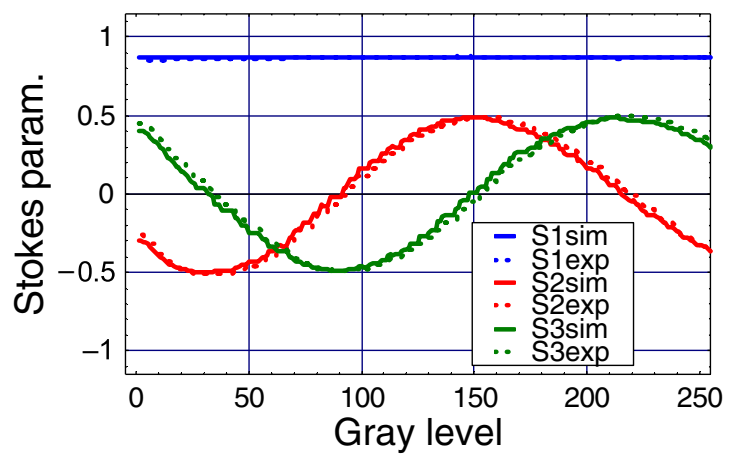

Fig. 3. Experimental and simulated values for the Stokes parameters, for input SOP linear at $+15^{\circ}$ and $\lambda=633 \mathrm{~nm}$. 


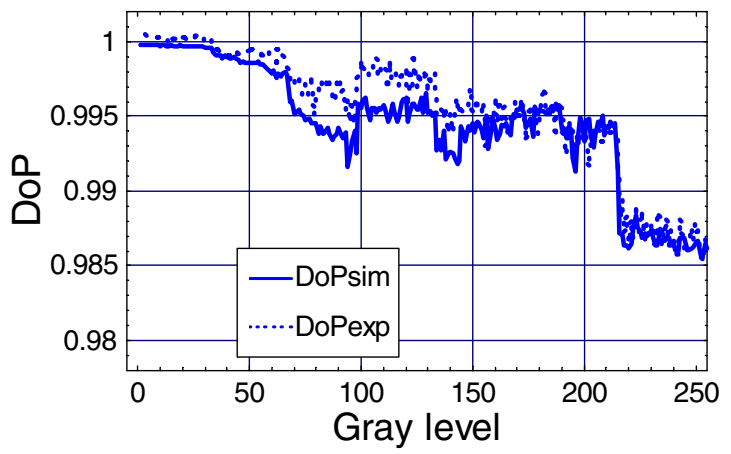

Fig. 4. Experimental and simulated DoP, for input SOP linear at $+15^{\circ}$ and $\lambda=633 \mathrm{~nm}$.

$S_{3}=1$ ). In Figs. $\underline{5}$ and $\underline{6}$ we show equivalent plots to the ones previously presented in Figs. $\underline{3}$ and 4 . Once again, we find very good agreement between experiment and simulation. In the present case, we note that the expression for the output SOP greatly simplifies

$$
\left\langle S_{\text {out }}\right\rangle=\left(\begin{array}{c}
1 \\
0 \\
-(\sin a / a) \sin \bar{\Gamma} \\
-(\sin a / a) \cos \bar{\Gamma}
\end{array}\right) .
$$

The DoP dependence turns out to be the same as for the input SOP used for calibration, i.e., Eq. (11). Circularly polarized light could also be a good option to be used for calibration purposes. However, it is more robust to generate linearly polarized light so that no additional polarization elements, such as a quarter wavelength waveplate, are necessary. We note that both configurations, linear at $+45^{\circ}\left(-45^{\circ}\right)$ and circular right-handed (left-handed), are the most sensitive input SOPs, since they provide the largest oscillation amplitude in the measurement of the third and fourth Stokes components, as can be seen in Figs. 1 and 5, together with the maximum variation of the Dop, shown in Fig. $\underline{6}$.

In conclusion, we have demonstrated a polarimetric method able to predict the performance of modern electro-optical devices showing retardance instabilities. We have extended the applicability of Stokes polarimetry to the characterization of these devices by a proper averaging process. This enables a fast and simple approach for full phase and flicker evaluation across the whole applied voltage range. From a more general perspective,

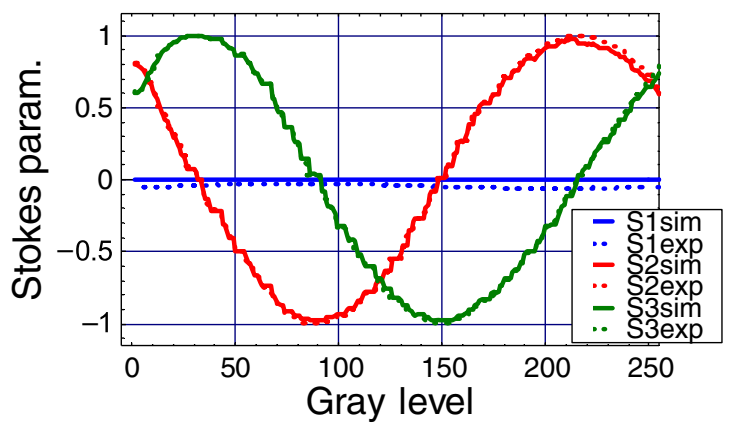

Fig. 5. Experimental and simulated values for the Stokes parameters, for input SOP right-handed circular and $\lambda=633 \mathrm{~nm}$.

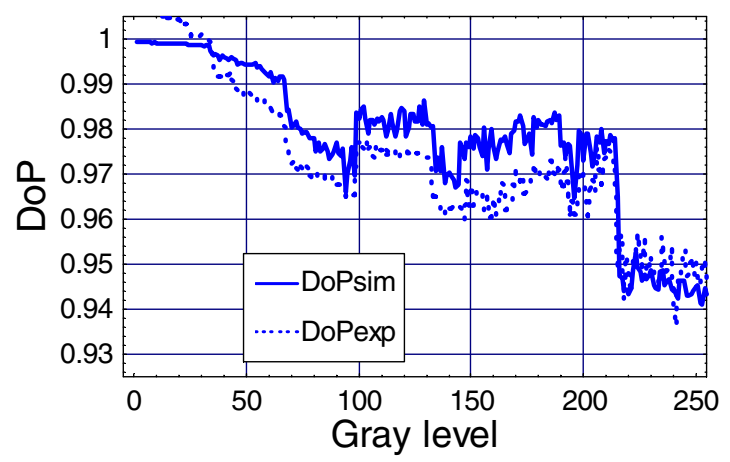

Fig. 6. Experimental and simulated DoP at output, for input SOP right-handed circular and $\lambda=633 \mathrm{~nm}$.

having a stochastic model for the polarization behavior may help, on one side, to refine the understanding of the dynamics of liquid crystal devices and, on the other side, to widen their applicability in polarization control, as with experiments dealing with unconventional polarization states [17].

Work supported by Min. de Trabajo y Competitividad of Spain (projects FIS2011-29803-C02-01 and FIS201129803-C02-02), by Gen. Valenciana of Spain (projects PROMETEO/2011/021 and ISIC/2012/013), and by Universidad de Alicante (project GRE12-14).

\section{References}

1. G. Goldstein, Polarized Light (Marcel Dekker, 2003).

2. A. De Martino, Y. K. Kim, E. Garcia-Caurel, B. Laude, and B. Drévillon, Opt. Lett. 28, 616 (2003).

3. A. Peinado, A. Lizana, J. Vidal, C. Iemmi, and J. Campos, Opt. Express 18, 9815 (2010).

4. S. T. Wu and D. K. Yang, Reflective Liquid Crystal Displays (Wiley, 2005).

5. N. Collings, T. Davey, J. Christmas, D. Chu, and B. Crossland, J. Disp. Technol. 7, 112 (2011).

6. C. Ramirez, E. Otón, C. Iemmi, I. Moreno, N. Bennis, J. M. Otón, and J. Campos, Opt. Express 21, 8116 (2013).

7. A. Márquez, C. Cazorla, M. J. Yzuel, and J. Campos, J. Mod. Opt. 52, 633 (2005).

8. J. E. Wolfe and R. A. Chipman, Appl. Opt. 45, 1688 (2006).

9. A. Hermerschmidt, S. Osten, S. Krüger, and T. Blümel, Proc. SPIE 6584, 65840E (2007).

10. J. R. Moore, N. Collings, W. A. Crossland, A. B. Davey, M. Evans, A. M. Jeziorska, M. Komarčević, R. J. Parker, T. D. Wilkinson, and $\mathrm{H}$. Xu, IEEE Photon. Technol. Lett. 20, 60 (2008).

11. A. Lizana, I. Moreno, A. Márquez, C. Iemmi, E. Fernández, J. Campos, and M. J. Yzuel, Opt. Express 16, 16711 (2008).

12. A. Lizana, I. Moreno, A. Márquez, E. Also, C. Iemmi, J. Campos, and M. J. Yzuel, Proc. SPIE 7442, 74420G-1 (2009).

13. J. García-Márquez, V. López, A. González-Vega, and E. Noé, Opt. Express 20, 8431 (2012).

14. A. Márquez, F. J. Martínez, S. Gallego, M. Ortuño, J. Francés, A. Beléndez, and I. Pascual, Proc. SPIE 8498, 84980L (2012).

15. C. Ramirez, B. Karakus, A. Lizana, and J. Campos, Opt. Express 21, 3182 (2013).

16. C. Flueraru, S. Latoui, J. Besse, and P. Legendre, IEEE Trans. Instrum. Meas. 57, 731 (2008).

17. T. G. Brown and Q. Zhan, Opt. Express 18, 10775 (2010). 